\title{
Bayesian parameter estimation in spectral quantitative photoacoustic tomography
}

\author{
Aki Pulkkinen ${ }^{\mathrm{a}}$, Ben T. Cox ${ }^{\mathrm{b}}$, Simon R. Arridge ${ }^{\mathrm{c}}$, Jari P. Kaipio ${ }^{\mathrm{a}, \mathrm{d}}$, and Tanja Tarvainen ${ }^{\mathrm{a}, \mathrm{c}}$ \\ ${ }^{a}$ Department of Applied Physics, University of Eastern Finland, P.O. Box 1627, FI-70211 \\ Kuopio, Finland \\ ${ }^{\mathrm{b}}$ Department of Medical Physics and Biomedical Engineering, University College London, \\ Gower Street, London WC1E 6BT, UK \\ ${ }^{c}$ Department of Computer Science, University College London, Gower Street, London WC1E \\ $6 \mathrm{BT}, \mathrm{UK}$ \\ ${ }^{d}$ Department of Mathematics, University of Auckland, Private Bag 92019, Auckland Mail \\ Centre, Auckland 1142, New Zealand
}

\begin{abstract}
Photoacoustic tomography (PAT) is an imaging technique combining strong contrast of optical imaging to high spatial resolution of ultrasound imaging. These strengths are achieved via photoacoustic effect, where a spatial absorption of light pulse is converted into a measurable propagating ultrasound wave. The method is seen as a potential tool for small animal imaging, pre-clinical investigations, study of blood vessels and vasculature, as well as for cancer imaging.

The goal in PAT is to form an image of the absorbed optical energy density field via acoustic inverse problem approaches from the measured ultrasound data. Quantitative PAT (QPAT) proceeds from these images and forms quantitative estimates of the optical properties of the target. This optical inverse problem of QPAT is illposed. To alleviate the issue, spectral QPAT (SQPAT) utilizes PAT data formed at multiple optical wavelengths simultaneously with optical parameter models of tissue to form quantitative estimates of the parameters of interest.

In this work, the inverse problem of SQPAT is investigated. Light propagation is modelled using the diffusion equation. Optical absorption is described with chromophore concentration weighted sum of known chromophore absorption spectra. Scattering is described by Mie scattering theory with an exponential power law. In the inverse problem, the spatially varying unknown parameters of interest are the chromophore concentrations, the Mie scattering parameters (power law factor and the exponent), and Grüneisen parameter. The inverse problem is approached with a Bayesian method. It is numerically demonstrated, that estimation of all parameters of interest is possible with the approach.
\end{abstract}

Keywords: multi-wavelength imaging, photoacoustics, inverse problems, image reconstruction

\section{INTRODUCTION}

Absorption of a short light pulse by soft tissue results in photoacoustic effect, where the absorbed energy is converted into an initial pressure distribution and a propagating acoustic wave. ${ }^{1-3}$ Photoacoustic tomography (PAT) attempts to form images of this initial pressure distribution, based on boundary measurements of the acoustic wave emitted via the photoacoustic effect. ${ }^{2,3}$ Quantitative photoacoustic tomography (QPAT) proceeds from the PAT reconstructions of the initial pressure distribution and seeks to deduce the optical properties of the target of interest, based on optical models of light propagation. ${ }^{2,3}$ One potential issue with QPAT, however, is that the absorbed optical energy density (the optical models) and the initial pressure distribution and the measured acoustic wave (the acoustical model) are connected via Grüneisen parameter, which in general is not

Further author information: (Send correspondence to A.P.)

A.P.: E-mail: Aki.Pulkkinen@uef.fi

Photons Plus Ultrasound: Imaging and Sensing 2016, edited by Alexander A. Oraevsky, Lihong V. Wang Proc. of SPIE Vol. 9708, 97081G - C 2016 SPIE · CCC code: 1605-7422/16/\$18 · doi: 10.1117/12.2205009 
known. Furthermore, estimation of the optical parameters (the quantification step) in QPAT is not only illposed, but can be non-unique as well. Spectral quantitative photoacoustic tomography (SQPAT) attempts to alleviate the issue by utilizing prior knowledge of the optical properties of tissue (as a function of wavelength) and measurements obtained at multiple optical wavelengths. ${ }^{4-10}$

In this work, the (optical) inverse problem in SQPAT is studied. The optical forward model, based on the diffusion approximation of the radiative transfer equation, ${ }^{11-13}$ is formulated in terms of spectral parameter models of optical absorption and scattering. The absorption is described by chromophore concentration weighted sum of known absorption spectra of the chromophores, whereas the scattering is described by using Mie scattering theory. The forward model is used to solve the inverse problem by using Bayesian framework. ${ }^{14-16}$ Parameters, that are estimated, are the chromophore concentrations, the Grüneisen parameter, and the Mie scattering theory based proportionality factor and exponent, all spatially varying.

\section{FORWARD MODEL}

The optical properties of biologial tissue typically depend on the optical wavelength. Within near-infrared wavelengths, the optical absorption can be expressed as $^{5-9}$

$$
\mu_{\mathrm{a}}(r, \lambda)=\sum_{n=1}^{N} c_{n}(r) \mu_{\mathrm{a}, n}(\lambda),
$$

where $r \in \Omega \subset \mathbb{R}^{k}$ is the position, $k$ is the number of spatial dimensions, $\lambda$ is the wavelength of light, $c_{n}$ $(n=1, \ldots, N)$ is the chromophore concentration of $n$th chromophore, and $\mu_{\mathrm{a}, n}$ is the absorption spectra of each chromophore. In this work, the concentrations $c_{n}$ should be interpreted as relative concentration with respect to the absolute absorption given by the absorption spectra $\mu_{\mathrm{a}, n}$; that is, if the absorption spectra is given by the absorption at molar concentration $\alpha$, then $c_{n}$ would correspond to molar concentration $c_{n} \alpha$. The optical (reduced) scattering follows the Mie scattering power-law

$$
\mu_{\mathrm{s}}^{\prime}(r, \lambda)=a(r) \lambda^{-b(r)}
$$

where $a(r)$ and $b(r)$ are the proportionality and exponent terms of the power-law. Equivalently, (2) can be expressed as

$$
\mu_{\mathrm{s}}^{\prime}(r, \lambda)=\mu_{\mathrm{s}, \mathrm{REF}}^{\prime}(r)\left(\frac{\lambda}{\lambda_{\mathrm{REF}}}\right)^{-b(r)}
$$

where $\mu_{\mathrm{s}, \mathrm{REF}}^{\prime}(r)$ is the optical scattering at reference wavelength $\lambda_{\mathrm{REF}}$, and it holds that $a(r)=\mu_{\mathrm{s}, \mathrm{REF}}^{\prime}(r) \lambda_{\mathrm{REF}}^{b(r)}$. Mie scattering power-law of form (3) is used for the rest of the paper.

With the spectral optical parameter models (1) and (3), the optical forward model of SQPAT can be expressed as

$$
p_{0}(r, \lambda)=\gamma(r) \mu_{\mathrm{a}}(r, \lambda) \Phi(r, \lambda),
$$

where $p_{0}$ is the (spatial) initial pressure distribution, $\gamma$ is the Grüneisen parameter, and $\Phi$ is the optical fluence, which is assumed here to be described by the optical diffusion equation ${ }^{11,12}$

$$
-\nabla \cdot \kappa(r, \lambda) \nabla \Phi(r, \lambda)+\mu_{\mathrm{a}}(r, \lambda) \Phi(r, \lambda)=0
$$

where $\kappa(r, \lambda)=\left(k\left(\mu_{\mathrm{a}}(r, \lambda)+\mu_{\mathrm{s}}^{\prime}(r, \lambda)\right)\right)^{-1}$ with the boundary condition

$$
\zeta_{k} \Phi(r, \lambda)+\frac{1}{2} \kappa(r, \lambda) \nabla \Phi(r, \lambda) \cdot \nu=s(r, \lambda)
$$

where $\zeta_{k}$ is dimensionality $k$ related parameter $\left(\zeta_{2}=\pi^{-1}, \zeta_{3}=4^{-1}\right), \nu$ is the unit normal on the boundary, and $s$ describes the inward light current (light source) on the boundary.

In practice, observations of $p_{0}$ in SQPAT are obtained after performing an acoustic reconstruction from the measured time domain acoustic boundary data. These observations, on the other hand, are obtained using some 
discrete wavelengths, and some spatial illumination pattern. Therefore, the forward model (4) can be expressed as

$$
p_{0, m}(r):=p_{0}\left(r, \lambda_{m}\right)=\gamma(r) \mu_{\mathrm{a}}\left(r, \lambda_{m}\right) \Phi_{m}\left(r, \lambda_{m}\right),
$$

where $m=1, \ldots, M$ denotes the measurement index, $\lambda_{m}$ is the wavelength used to obtain measurement $m$, and $\Phi_{m}$ is the fluence resulting from illumination with light source $s_{m}$.

In this work, the forward model (7) is discretized by using finite element method (FEM), into grid nodes $r_{q}$ $(q=1, \ldots, Q)$, resulting in

$$
p_{0, m}\left(r_{q}\right)=\gamma\left(r_{q}\right) \mu_{\mathrm{a}}\left(r_{q}, \lambda_{m}\right) \Phi_{m}\left(r_{q}, \lambda_{m}\right), \quad m=1, \ldots, M, \quad q=1, \ldots, Q,
$$

which can equivalently be written as

$$
y^{\prime}=f(x)
$$

where $y^{\prime} \in \mathbb{R}^{M Q}$ and $f(x): \mathbb{R}^{(N+3) Q} \rightarrow \mathbb{R}^{M Q}$ correspond to left and right hand sides of (8) respectively, and $x$ is the collection of parameters of interest in SQPAT: chromophore concentrations $c_{1}, \ldots, c_{N}$, Grüneisen parameter $\gamma$, and the Mie scattereing parameters $\mu_{\mathrm{s}, \mathrm{REF}}^{\prime}$ and $b$, each spatially varying. For details on the numerical implementation, see. ${ }^{10}$

\section{BAYESIAN INVERSION}

In this work, the estimation of the parameters of interest is done following the Bayesian framework. ${ }^{14-16}$ Observation model for an additive error model is

$$
y=f(x)+e,
$$

where $y \in \mathbb{R}^{M Q}$ is the fixed (noisy) observation polluted with noise $e \in \mathbb{R}^{M Q}$. Let $p(\cdot)$ denote probability density function of a random variable. It follows, from the observation model (10), that the conditional distribution of $y$ for a given $x$ independent of $e$ is

$$
p(y \mid x)=p_{e}(y-f(x)),
$$

where $p_{e}$ is the probability density function of the noise $e$. Equation (11) is called the likelihood function. The Bayes' theorem states that

$$
p(y \mid x) p(x)=p(x \mid y) p(y) .
$$

Given an observation (sample) $y$, which is a fixed vector, $p(y)$ is a constant and it follows from the Bayes' theorem that

$$
p(x \mid y) \propto p(y \mid x) p(x) .
$$

Substituting the likelihood function (11), and incorporating all the prior knowledge of the unknown parameter $x$ into $p(x)=p_{x}(x)$, the posterior density function is found to be

$$
p(x \mid y) \propto p_{e}(y-f(x)) p_{x}(x) .
$$

Assuming that the noise $e$ follows normal distribution and that we choose the prior information of $x$ to be described by a normal distribution as well, the posterior density function (14) can be expressed as*

$$
p(x \mid y) \propto \exp \left(-\frac{1}{2} u(x, y)\right)
$$

where

$$
u(x, y)=\left\|L_{e}\left(y-f(x)-\eta_{e}\right)\right\|^{2}+\left\|L_{x}\left(x-\eta_{x}\right)\right\|^{2},
$$

${ }^{*}$ For some random vector $z \in \mathbb{R}^{N}$ that follows the normal distribution, such that $z \sim \mathcal{N}\left(\eta_{z}, \Gamma_{z}\right)$, the probability density function is

$$
p_{z}(z)=\left((2 \pi)^{N}\left|\Gamma_{z}\right|\right)^{-1 / 2} \exp \left(-\left.\frac{1}{2}|| L_{z}\left(z-\eta_{z}\right)\right|^{2}\right)
$$

where $\left|\Gamma_{z}\right|$ is the determinant of the covariance matrix, and $L_{z}^{\top} L_{z}=\Gamma_{z}^{-1}$ is the Cholesky decomposition of the inverse covariance matrix. 
and $e \sim \mathcal{N}\left(\eta_{e}, \Gamma_{e}\right), x \sim \mathcal{N}\left(\eta_{x}, \Gamma_{x}\right)$ with $L_{e}^{\top} L_{e}=\Gamma_{e}^{-1}$ and $L_{x}^{\top} L_{x}=\Gamma_{x}^{-1}$. The posterior density function describes the statistical knowledge of $x$ as inferred by a given observation $y$. From the posterior distribution, it is possible to obtain, for example, credibility intervals, and point estimates. The point estimate that is used in this work is the maximum a posteriori (MAP) estimate, which can be shown to be

$$
x_{\mathrm{MAP}}=\underset{x}{\arg \min } u(x, y) .
$$

In this work, MAP estimates are obtained by using Gauss-Newton iteration.

In this work, the prior that is used is the Ornstein-Uhlenbeck prior, ${ }^{10,17,18}$ defined for spatially varying parameter $z$ at grid nodes $r_{q}$ via identity covariance matrix $\Xi$ as

$$
\Gamma_{z}=\sigma_{z}^{2} \Xi, \quad\{\Xi\}_{i j}=\exp \left(-\left\|r_{i}-r_{j}\right\| / \xi\right), \quad i, j=1, \ldots, Q,
$$

where $z \in\left\{c_{1}, \ldots, c_{N}, \gamma, \mu_{\mathrm{s}, \mathrm{REF}}^{\prime}, b\right\}, z \sim \mathcal{N}\left(\eta_{z}, \Gamma_{z}\right)$, and $\xi$ is the characteristic length scale. The OrnsteinUhlenbeck prior descibes a spatial parameter distribution, that has exponentially decaying spatial covariance as a function of distance between two points. Thus the prior promotes estimates that have some level of spatial structure in them, as is reasonable to expect in biomedical applications. For each of the estimated parameters, $\eta_{z}$ and $\sigma_{z}$ were chosen as

$$
\eta_{z}=\frac{1}{2}(\max z+\min z), \quad \sigma_{z}=\frac{1}{2}(\max z-\min z),
$$

where $\max z$ and $\min z$ are the maximum and minimum values that $z$ is assumed to vary between. This choice corresponds to expecting the unknown values to reside in the range [min $z, \max z]$ with probability of $68.3 \%$ and supporting values outside the expected range as well. The combined prior can thus be expressed as $x \sim \mathcal{N}\left(\eta_{x}, \Gamma_{x}\right)$ with

$$
\eta_{x}=\left(\eta_{c_{1}}, \ldots, \eta_{c_{N}}, \eta_{\gamma}, \eta_{\mu_{\mathrm{s}, \mathrm{REF}}^{\prime}}, \eta_{b}\right), \quad \Gamma_{x}=\operatorname{Diag}\left\{\Gamma_{c_{1}}, \ldots, \Gamma_{c_{N}}, \Gamma_{\gamma}, \Gamma_{\mu_{\mathrm{s}, \mathrm{REF}}^{\prime}}, \Gamma_{b}\right\}
$$

\section{SIMULATIONS}

In this work, the studied geometry is a two dimensional circle with radius of $5 \mathrm{~mm}$ and center at origin. Total of six illuminations (two illuminations at three distinct wavelengths) are used in the forward model:

$$
\begin{array}{ll}
s_{1}(r)=s\left(r, \lambda_{1}\right)=\left|r_{1}\right| / 5 \mathrm{~mm}, & s_{2}(r)=s\left(r, \lambda_{1}\right)=\left|r_{2}\right| / 5 \mathrm{~mm}, \\
s_{3}(r)=s\left(r, \lambda_{2}\right)=\left|r_{1}\right| / 5 \mathrm{~mm}, & s_{4}(r)=s\left(r, \lambda_{2}\right)=\left|r_{2}\right| / 5 \mathrm{~mm}, \\
s_{5}(r)=s\left(r, \lambda_{3}\right)=\left|r_{1}\right| / 5 \mathrm{~mm}, & s_{6}(r)=s\left(r, \lambda_{3}\right)=\left|r_{2}\right| / 5 \mathrm{~mm},
\end{array}
$$

where $r=\left(r_{1}, r_{2}\right)$ with $r_{1}$ and $r_{2}$ being the horizontal and vertical coordinates, $|\cdot|$ is the absolute value, and the wavelengths are $\lambda_{1}=700 \mathrm{~nm}, \lambda_{2}=800 \mathrm{~nm}, \lambda_{3}=900 \mathrm{~nm}$. Illuminations $s_{1}, s_{3}$, and $s_{5}$ describe a light source originating simultaneously from the left and right of the target domain, and illuminations $s_{2}, s_{4}$, and $s_{6}$ a source originating from top and bottom.

The parameters $\left(c_{1}, \ldots, c_{N}, \gamma, \mu_{\mathrm{s}, \mathrm{REF}}^{\prime}, b\right)$ of the target were chosen such that they are in biologically relevant magnitude. The target was composed of $N=3$ chromophores: fat $\left(c_{1}\right)$, deoxygenated $\left(c_{2}\right)$ and oxygenated $\left(c_{3}\right)$ blood. The absorption spectra used for the chromophores, at the used wavelengths, are shown in Table 1. Two sets of parameters were studied: smoothly varying parameters and parameters with sharp boundaries. The parameters used to generate the simulated data (virtual measurements) are shown in Figure 1.

After simulating the data in a triangulated mesh with $Q=4480$ grid nodes, the data was interpolated to a lower density triangulated mesh with $Q=3027$ grid nodes and noise was added to it. Interpolation was done in order to avoid inverse crime during the estimation. The noise, that was added, was drawn from a normal distribution with zero mean and for each illumination $s_{m}$ had standard deviation of $\sigma_{m}=0.001 \max _{q} p_{0, m}\left(r_{q}\right)$. The data corresponding to the parameters is shown in Figure 2.

The MAP estimates (17) were obtained by using a Gauss-Newton algorithm augmented with a line search. For the noise statistics, accurately characterized parameters were assumed with $\eta_{e}=0$ and $\Gamma_{e}=\operatorname{Diag}\left\{\sigma_{1}^{2}, \ldots, \sigma_{6}^{2}\right\}$. For the prior, the Ornstein-Uhlenbeck was used, as defined in (20), with $\xi=1 \mathrm{~mm}$. The MAP estimates of 
Table 1. Absorption spectra used in this paper: $\mu_{\mathrm{a}, 1}$ corresponds to fat, ${ }^{19} \mu_{\mathrm{a}, 2}$ and $\mu_{\mathrm{a}, 3}$ to deoxygenated and oxygenated blood at physiologically realistic molar concentrations. ${ }^{20}$

\begin{tabular}{cccc}
\hline \hline$\lambda(\mathrm{nm})$ & $\mu_{\mathrm{a}, 1}(\lambda)\left(\mathrm{mm}^{-1}\right)$ & $\mu_{\mathrm{a}, 2}(\lambda)\left(\mathrm{mm}^{-1}\right)$ & $\mu_{\mathrm{a}, 3}(\lambda)\left(\mathrm{mm}^{-1}\right)$ \\
\hline 700 & 0.0700 & 0.9781 & 0.1713 \\
800 & 0.0750 & 0.4496 & 0.4632 \\
900 & 0.0800 & 0.4754 & 0.7155 \\
\hline \hline
\end{tabular}
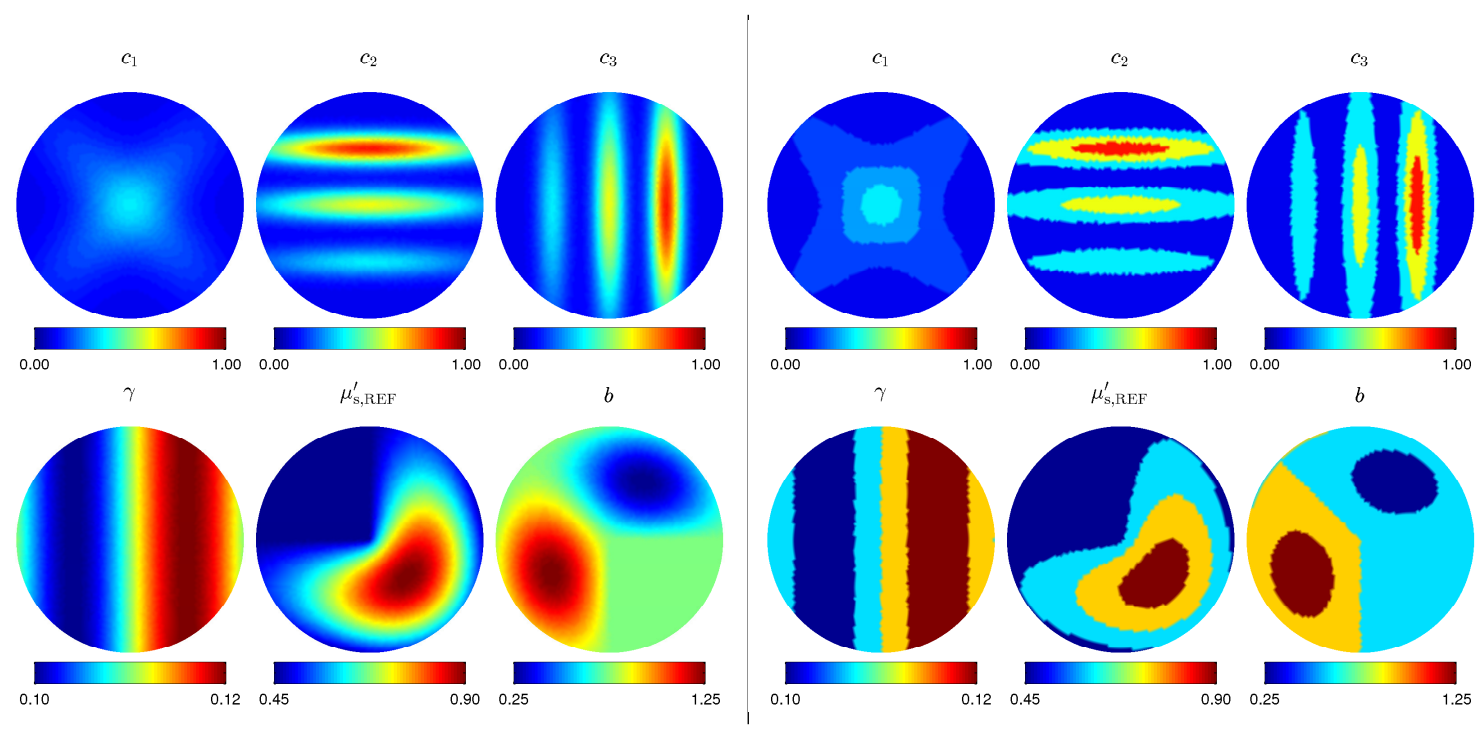

Figure 1. Smoothly (on the left) and sharply (on the right) varying parameters used to generate the simulated data.

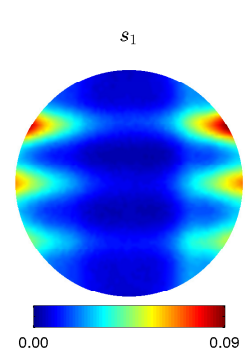

$s_{2}$

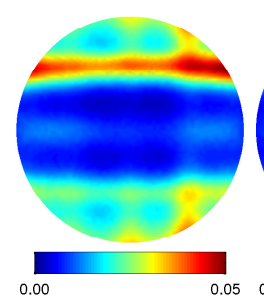

$s_{3}$
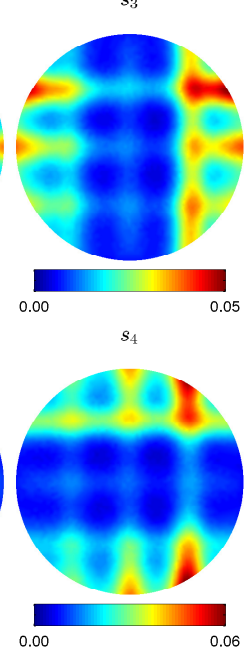

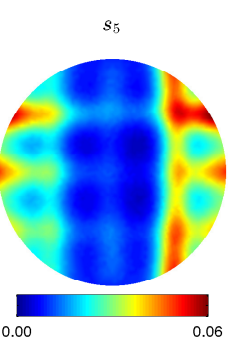

$s_{6}$

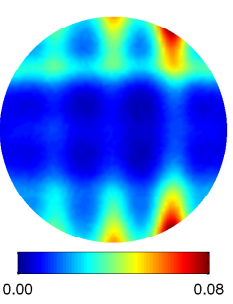

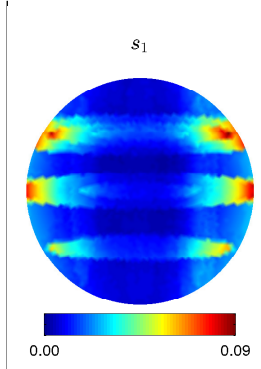
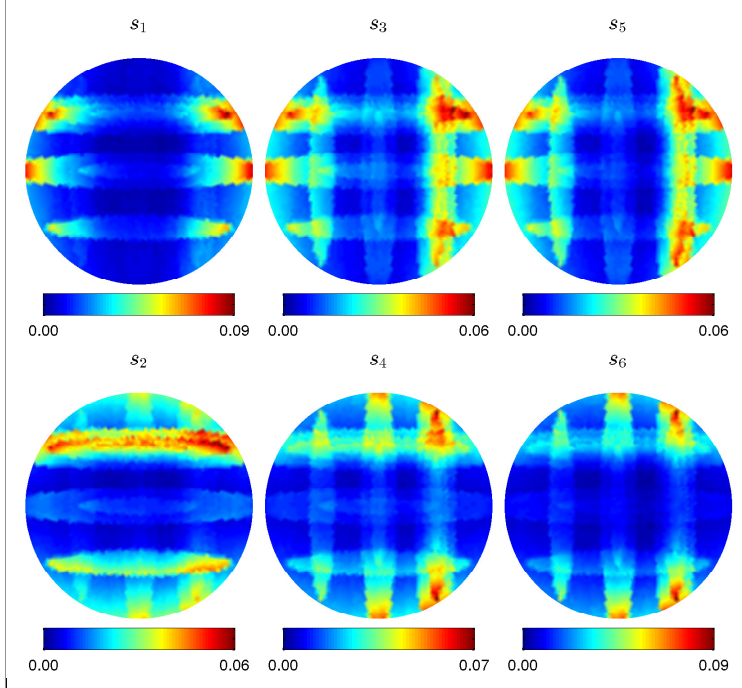

$s_{4}$

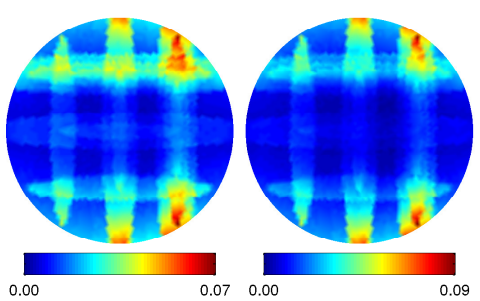

Figure 2. Simulated data ( $p_{0, m}$ as corrupted by noise) computed with smoothly (on the left) and sharply (on the right) varying parameters. The illumination (21), corresponding to each data, is marked in the figure.

$c_{1}, c_{2}, c_{3}, \gamma, \mu_{\mathrm{s}, \mathrm{REF}}^{\prime}, b$ are shown in Figure 3 and their relative errors are shown in Table 2. Relative errors were computed using

$$
E=\frac{\left\|z_{\mathrm{MAP}}-z_{\mathrm{TRUE}}\right\|}{\left\|z_{\mathrm{TRUE}}\right\|},
$$



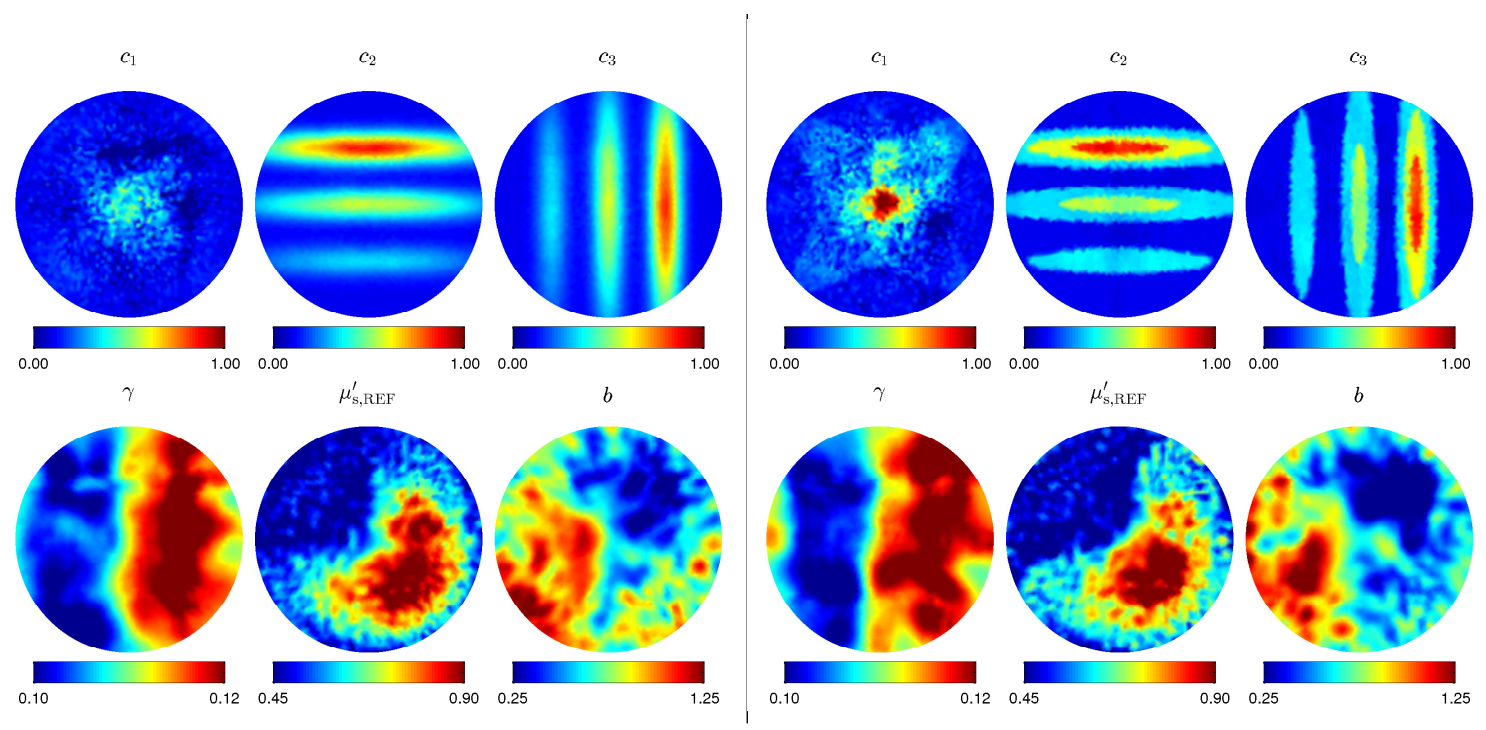

Figure 3. MAP estimates computed with data that had smoothly (on the left) and sharply (on the right) varying parameters.

Table 2. Relative errors of the MAP estimates for the smoothly and sharply varying parameters studied.

\begin{tabular}{rrr}
\hline \hline Parameter & Smooth & Sharp \\
\hline$c_{1}$ & $43.3 \%$ & $68.4 \%$ \\
$c_{2}$ & $3.5 \%$ & $6.1 \%$ \\
$c_{2}$ & $3.4 \%$ & $6.7 \%$ \\
$\gamma$ & $2.2 \%$ & $2.9 \%$ \\
$\mu_{\mathrm{s}, \mathrm{REF}}^{\prime}$ & $7.6 \%$ & $8.5 \%$ \\
$b$ & $18.9 \%$ & $22.5 \%$ \\
\hline \hline
\end{tabular}

where $z_{\mathrm{MAP}}$ is the MAP estimate of any of $c_{1}, c_{2}, c_{3}, \gamma, \mu_{\mathrm{s}, \mathrm{REF}}^{\prime}, b$, and $z_{\mathrm{TRUE}}$ is the respective true parameter value, and $\|\cdot\|$ is the $l^{2}$-norm of the discretized vector.

The MAP estimates, shown in Figure 3, demonstrate that the approach can be used to quantitatively (and thus qualitatively) estimate all the parameters of interest $\left(c_{1}, \ldots, c_{N}, \gamma, \mu_{\mathrm{s}, \mathrm{REF}}^{\prime}, b\right)$. The estimate of fat $\left(c_{1}\right)$ is evidently poor due to its low optical absorption in comparison to blood $\left(c_{2}\right.$ and $\left.c_{3}\right)$. However, when the true $c_{1}$ was sharply varying, the estimates resemblance to the true parameter especially away from the center of the domain is evident, whereas the largest differences are found at the center of the domain. This is, at least partially, explained by the fact that the center of the domain has the poorest quality data coming from for all the illuminations due to the low fluence. Both of the blood parameters $\left(c_{2}\right.$ and $\left.c_{3}\right)$ are well resolved with smoothly and sharply varying parameters. The Grüneisen parameter $(\gamma)$, and the scattering parameters $\left(\mu_{\mathrm{s}, \mathrm{REF}}^{\prime}\right.$ and $\left.b\right)$ are not estimated as sharply as the blood parameters, but their estimates still resemble the true parameters. The relative errors, shown in Table 2, show that the sharply varying parameters are all estimated to lower accuracy than the smoothly varying parameters.

\section{CONCLUSION}

A forward model for the spectral quantitative photoacoustic tomography based on the diffusion approximation of the optical fluence was presented. Chromophore concentration weighted sum was used to describe the optical absorption as a function of wavelength, whereas Mie scattering theory was used for the reduced scattering. The model was used in the Bayesian framework to form estimates of two dimensional targets with parameter scales in biologically relevant regime. The parameters of interest, the chromophore concentrations, Grüneisen, and the 
Mie scattering proportionality and exponent terms, all spatially varying, were successfully estimated using the described approach.

The presented approach enables inclusion of quantitative prior information into the estimation process in SQPAT. This is important, as the inverse problem in SQPAT is ill-posed, which can render the problem numerically unstable. Furthermore, the Bayesian approach permits inclusion of the statistical information of noise and uncertainties present in the measurement setup into the estimation. Although, only MAP estimates were inspected in this work, the Bayesian approach provides the entire posteriori distribution of the unknowns as a solution to the inverse problem. In addition to MAP estimates, error of the estimates in the form of e.g. credibility intervals can also be computed.

\section{ACKNOWLEDGMENTS}

This work has been supported by the Academy of Finland (projects 136220, 272803, 286247, and 250215 Finnish Centre of Excellence in Inverse Problems Research).

\section{REFERENCES}

[1] Gusev, V. Z. and Karabutov, A. A., [Laser Optoacoustics], American Institute of Physics (1992).

[2] Li, C. and Wang, L. V., "Photoacoustic tomography and sensing in biomedicine," Phys. Med. Biol. 54, R59-R97 (2009).

[3] Beard, P., "Biomedical photoacoustic imaging," Interface Focus 1, 602-631 (2011).

[4] Razansky, D., Vinegoni, C., and Ntziachristos, V., "Multispectral photoacoustic imaging of fluorochromes in small animals," Opt. Lett. 32(19), 2891-2893 (2007).

[5] Razansky, D., Distel, M., Vinegoni, C., Ma, R., Perrimon, N., Köster, R. W., and Ntziachristos, V., "Multispectral opto-acoustic tomography of deep-seated fluorescent proteins in vivo," Nature Photon. 3, 412-417 (2009).

[6] Yuan, Z. and Jiang, H., "Simultaneous recovery of tissue physiological and acoustic properties and the criteria for wavelength selection in multispectral photoacoustic tomography," Opt. Lett. 34(11), 1714-1716 (2009).

[7] Laufer, J., Cox, B., Zhang, E., and Beard, P., "Quantitative determination of chromophore concentrations from 2D photoacoustic images using a nonlinear model-based inversion scheme," Appl. Optics 49, 1219-1233 (2010).

[8] Cox, B. T., Arridge, S. R., and Beard, P. C., "Estimating chromophore distributions from multiwavelength photoacoustic images," J. Opt. Soc. Am. A 26, 443-455 (2009).

[9] Bal, G. and Ren, K., "On multi-spectral quantitative photoacoustic tomography in diffusive regime," Inv. Probl. 28, 025010 (2012).

[10] Pulkkinen, A., Cox, B. T., Arridge, S. R., Kaipio, J. P., and Tarvainen, T., "A Bayesian approach to spectral quantitative photoacoustic tomography," Inv. Probl. 30, 065012 (2014).

[11] Case, K. M. and Zweifel, P. F., [Linear Transport Theory], Addison-Wesley (1967).

[12] Ishimaru, A., [Wave Propagation and Scattering in Random Media Vol 1,], Academic Press (1978).

[13] Tarvainen, T., Cox, B. T., Kaipio, J. P., and Arridge, S. R., "Reconstructing absorption and scattering distributions in quantitative photoacoustic tomography," Inv. Probl. 28, 084009 (2012).

[14] Tarantola, A., [Inverse problem theory and methods for model parameter estimation], SIAM Society for Industrial and Applied Mathematics, Philadelphia (2005).

[15] Kaipio, J. and Somersalo, E., [Statistical and Computational Inverse Problems], Springer, New York (2005).

[16] Kaipio, J. and Somersalo, E., "Statistical inverse problems: discretization, model reduction and inverse crimes," J. Comput. Appl. Math 198, 493-504 (2007).

[17] Rasmussen, C. E. and Williams, C. K. I., [Gaussian Processes for Machine Learning], The MIT Press (2006).

[18] Pulkkinen, A., Cox, B. T., Arridge, S. R., Kaipio, J. P., and Tarvainen, T., "Quantitative photoacoustic tomography using illuminations from a single direction," J. Biomed. Opt. 20(3), 036015 (2015). 
[19] Cheong, W. F., Prahl, S. A., and Welch, A. J., "A review of the optical properties of biological tissues," IEEE J. Quantum Electronics 26, 2166-2185 (1990).

[20] Cope, M., The application of near infrared spectroscopy to non invasive monitoring of cerebral oxygenation in the newborn infant, $\mathrm{PhD}$ thesis, University of London (1991). 